\title{
An In Vitro Patient-Tailored Model of Human Cerebral Artery for Simulating Endovascular Intervention
}

\author{
Seiichi Ikeda ${ }^{1}$, Fumihito Arai ${ }^{1}$, Toshio Fukuda ${ }^{1}$, Makoto Negoro $^{2}$, \\ Keiko Irie ${ }^{2}$, and Ikuo Takahashi ${ }^{3}$ \\ ${ }^{1}$ Dept. of Micro-Nano Systems Engineering, Nagoya Iniverisity, \\ Furo-cho 1, Chikusa-ku, Nagoya, 464-8603, Japan \\ ikeda@robo.mein.nagoya-u.ac.jp, \\ \{arai, fukuda\}@ mein.nagoya-u.ac.jp, \\ http: / /www.mein.nagoya-u.ac.jp/index.html \\ ${ }^{2}$ Dept. of Neurosurgery, Fujita Health University, \\ Kutsukake-cho, Toyoake, 470-1192, Japan \\ ${ }^{3}$ Dept. of Neurosurgery, Anjo Kosei Hospital, \\ Anjo-cho 28, Anjo, 446-8602, Japan
}

\begin{abstract}
An in vitro patient-tailored reproduction model of cerebral artery, a hardware platform for simulating endovascular intervention for making diagnoses and surgical trainings is presented. 3-D configuration of vessel lumen is reproduced as vessel model with $13 \mu \mathrm{m}$ modeling resolution, using CT and MRI information. Physical characteristics of cerebral artery, such as elastic modulus and friction coefficient, are also reproduced. We also propose a novel method to visualize stress condition on vessel wall using photoelastic effect. Consequently, it should be helpful for clinical applications, academic researches and other various purposes.
\end{abstract}

\section{Introduction}

Recently established endovascular intervention is a potent treatment modality for various vascular diseases, which reduces the invasiveness for patients [1][2]. Many devices for endovascular intervention have been developed since late 1980 to treat with various diseases. However, the tortuosity and diversity of cerebral vasculature disturbs maneuvering of those sophisticated devices, so it requires an advanced surgical skill and experience. However current training environment for this surgery is inadequate, and this situation makes surgical skills more difficult to acquire [5].

Traditionally, interventionalists have solely depended on images projected on displays or papers as an only means to provide medical information obtained by angiography or other imaging modalities. Although, it is now possible to reconstruct the 3 -D configuration of vascular system from 2-D fluoroscopic images, even so, accurate recognition of 3-D vascular configuration is still not easy and it may lead to misinterpret information.

As a solution for these difficulties, we propose an in vitro vessel model of cerebral artery that reproduces the 3-D configuration of patient's vascular lumens as membra- 
nous silicone structure, utilizing CT and MRI information. With this model, it becomes possible for young trainees to practice surgical skills of endocvascular intervention. And it becomes also possible with this model to preliminary simulate neurovascular procedures before surgery.

So far, dissection based modeling method for vasculature, (a method that utilize vessel as casting mold) was developed by P. Guilloud et al. And, this method is now largely utilized for various purposes [3][4]. However, the dissection model is not applicable for preliminary simulation and requires rather high time cost and labor cost for respective modeling.

\section{Required Condition for Surgical Simulator}

\subsection{Patient-Tailored High Precision Modeling}

Cerebral artery and cerebrovascualr disease take very different configuration for respective patients (Fig.1), and the diversity of configuration contributes to make neurovascular procedures more difficult. Therefore conventional surgical simulator which imitates the general vascular configuration is inadequate for comprehensive surgical training. Here, it becomes very helpful to construct a patient-tailored vessel model using CT and MRI information which are usually available for all patient. Furthermore, as a simulator for neurovascular procedures, vessel lumen with less than $1 \mathrm{~mm}$ in diameter need be reproduced

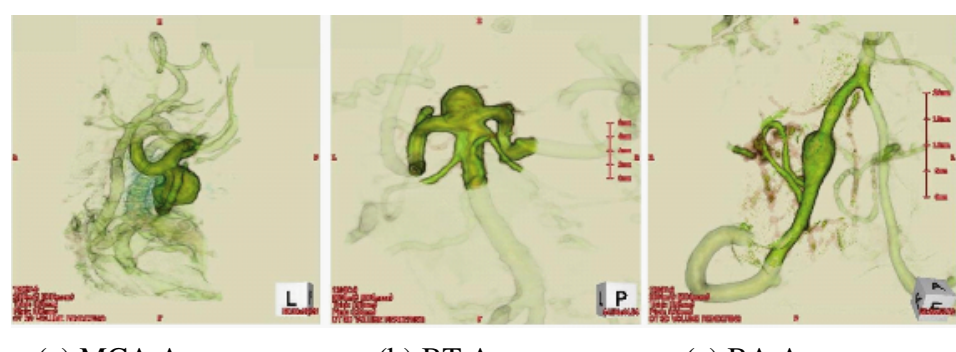

(a) MCA Aneurysm

(b) BT Aneurysm

(c) BA Aneurysm

Fig. 1. Some typical configuration of cerebral aneurysm

\subsection{Reproduction of Physical Characteristics}

Operational feel plays an important role in treating cerebrovascular diseases safely without applying excessive piercing stress on vessel structure. Therefore, reproduction of physical characteristics of vesculature becomes important, to reproduce the difficulties of manipulating medial instruments and the behaviors of these medical instruments (e.g. slip / stick motion and unwinding motion of catheter) Meanwhile, vessel model should be compatible with medial imaging modalities (especially DAS), to allow simulating neurovascular procedures under practical IVR (interventional radiology) condition. 


\subsection{Reproduction of Visco-Elastic Vascular Deformation}

Membranous vessel configuration and surrounding soft brain tissue allow cerebral artery to deform against surgical treatments, and some neurovasclar treatments affirmatively utilize this characteristics. Expansion of stenosis with balloons and stents is an instance of this treatment. Meanwhile, this characteristics simultaneously results in technical difficulties. Therefore, to reproduce these important features, reproduction of visco-elastic deformation becomes essential

\subsection{Summary of Required Conditions}

As the required conditions for an in vitro vascularture model for simulating neurovascular procedures, we summarize the above contents as follows;

1) Patient-tailored with sub-millimeter resolution

2) Reproduced with thin membranous vacular configuration

3) Reproduced with material properties of vascular tissue

4) Reproduced with visco-elastic vascular deformation

5) Compatible with medical imaging modalities

\section{An In Vitro Cerebral Arterial Model Reproduced with Human- Like Physical Characteristics}

\subsection{Rapid Prototyping for Patient-Tailored Modeling}

Lately developed laminating modeling modality (rapid prototyping (RP) modality) has made it possible to construct organ models based on individual information, such as CT and MRI information. This RP-based organic model had proved its effectiveness for anatomical studies and for some clinical applications.

However, since the material used in RP modeling is limited, it is currently impossible to express the physical characteristics of organic tissues (current RP model is too rigid, fragile and anisotropic). Furthermore the RP modeling is usually accompanied tiered up rough surface (disturbs manipulating medical instruments, and detariorates visibility). Consequently, the direct PR modeling modality is still not adequate for organic reproduction.

\subsection{Production Methodology for Patient-Tailored Modeling}

Here in after, we introduce a production method for an in vitro patient-tailored vasculature model that satisfies all the requirements described. Proposed production modality consists of the following 4 technical procedures; (1) reconstruction of 3-D vascular configuration, (2) fabrication of solid lumen model with RP modality, (3) fabrication of thin silicone membrane and (4) hollow construction of vascular lumen.

Firstly, we reconstructed the 3-D configuration of cerebral artery, using fluoroscopic image obtained with CT angiography (resolution: $0.3 \mathrm{~mm}$, imaging pitch: $0.5 \mathrm{~mm}$ ). We reconstructed the 3-D field of imaged area by heaping up these 2-D images, then extracted 3-D surface of cerebral artery by extracting area with identical CT value. Then we eliminated unimportant branches and discontinuous segment from this structure to 
simplify to leave only basilar artery and aneurysm. By this means, we finally obtained a 3-D structure of basilar tip artery from the CT information (Fig. 2 (a)).

Then we fabricated solid structure of the reconstructed vascular lumen by RP modality (Fig. 2 (b)). Laminating thickness was $13 \mu \mathrm{m}$. We adopted fused deposition RP modality, since it allows the use of sulfonamide resin as construction material. Its material characteristics (melts at low temperature into high fluidity, easily dissolves in acetone) are considerably favorable in following modeling process.

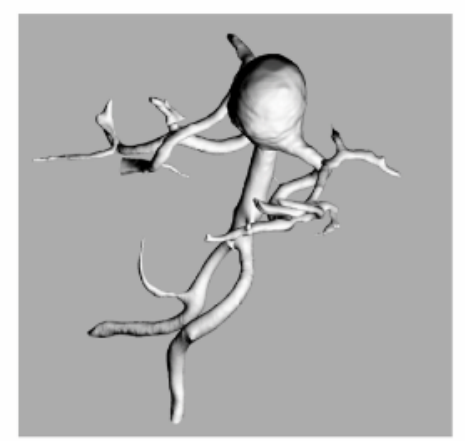

(a) Reconstructed 3D figure of basilar artery reconstructed with CT angiography

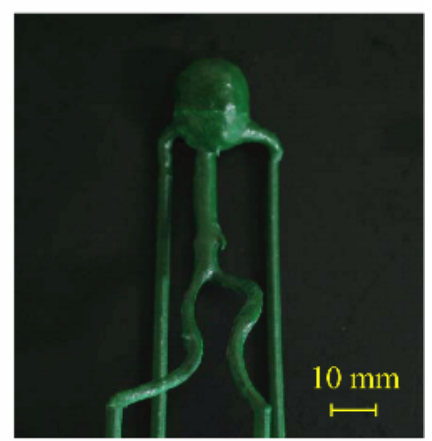

(b) Solid lumen structure fabricated by RP modality (layering pitch: $13 \mu \mathrm{m}$ )

Fig. 2. Materialization of individual vasculature structure based on CT / MRI angiography (small uninterested branches are ablated)

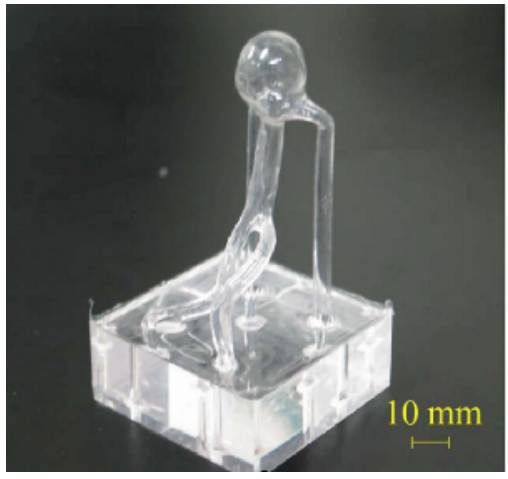

Fig. 3. Elastic membranous model of basilar artery fabricated based on individual information (inner RP model is eliminated)

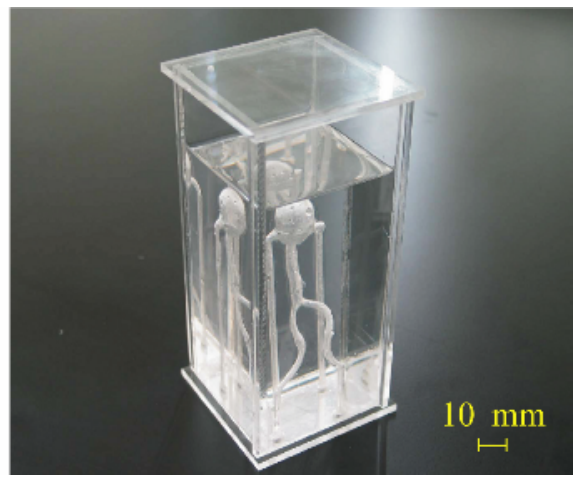

Fig. 4. Cerebral arterial model with vascularlike membranous configuration and brainlike soft circumferential configuration

We fabricated thin uniform membrane of silicone elastomer (provides artery-like physical characteristics and good transparency) with $600 \mu \mathrm{m}$ thick around this RP lumen structure, through velocity controlled dipping coating process and following addi- 
tion polymerization process (Fig. 3; in this figure, solid lumen model is eliminated). After that, we embedded the structure within silicone gel (provides brain like elastic modulus) inside a cubic casting mold to reproduce circumferential soft brain structure.

Then we eliminated inward RP lumen structure through two independent processes. Firstly, we evacuated most part of the lumen model by selectively melting the RP structure at $120^{\circ} \mathrm{C}$ (higher than the melting point of RP model and lower than the heatproof temperature of silicone). Then we completely dissolved the remained quantity (especially in tight corners and dead ends) by injecting acetone inside lumens. With this procedure, the evacuation was managed in short period.

Consequently, we fabricated a transparent silicone model of basilar artery reproduced with thin membranous configuration and surrounding soft brain configuration that hollowly reproduces the vascular lumen of living patient with $13 \mu \mathrm{m}$ modeling resolution (Fig. 4). As the reflection indexes of silicone elastomer and silicone gel are almost identical value (silicone elastomer $\left(\mathrm{n}_{\mathrm{D}}{ }^{25}\right): 1.410$, silicone gel $\left.\left(\mathrm{n}_{\mathrm{D}}{ }^{25}\right): 1.404\right)$, no optical distortion take place and it realized good visibility.

\section{APPLICATION: Photoelastic Stress Visualization and Analysis}

Transparent isotropic material, including silicone elastomer and polyurethane elastomer, shows temporal birefringent effect (double refraction) when external load is applied. And making use of this effect, we visualized stress distribution on proposed vessel model as fringe pattern (called photoelastic stress analysis). In this experiment, we applied transparent polyurethane elastomer (photoelastic coefficient: $3.5 \times 10^{-9} \mathrm{~Pa}^{-1}$; Young's modulus: $1.8 \mathrm{MPa}$ ) as construction material to increase its sensitivity to birefringent effect. And with a general configuration of circular polariscope, stress condition on vascular membrane was clearly visualized as rainbow-colored interference fringes pattern as shown in Fig. 5.

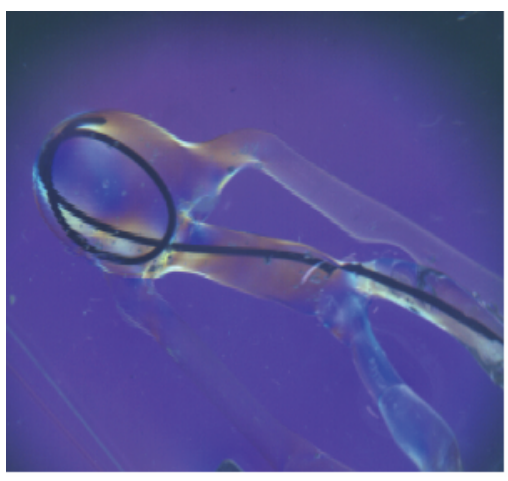

Fig. 5. Stress distribution against catheter operation visualized by means of photoelastic effect with circular polariscope

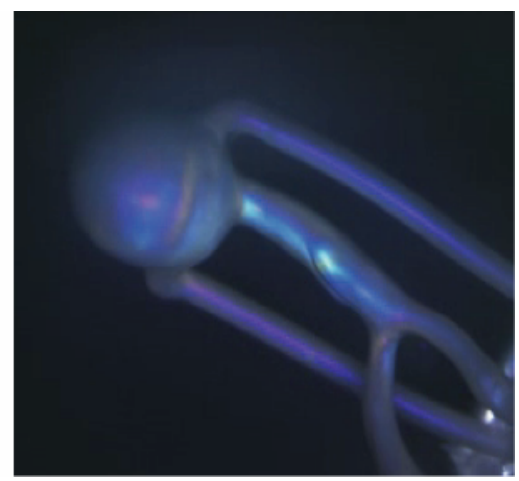

Fig. 6. Stress distribution against catheter operation visualized by means of photoelastic effect with reflective circular polariscope 
However, it is impossible, with this method, to distinguish the optical effect took place on fore vascular wall and rear wall. As a solution, we developed a new method for quantitative real-time stress analysis. In this method, projected polarized light, passing through fore wall with birefringent effect, is reflected at the surface of inner lumen filled with liquid added with aluminum powder (serves as mirror), then passes back the same point on fore wall with doubled birefringent effect (sensitivity becomes doubled than usual). Fig. 6 shows the visualized stress pattern with this method. As to this method, observed color can be related with phase shift $\boldsymbol{R}$, and stress condition (max. principal stress $\sigma_{1}$, min. principal stress $\boldsymbol{\sigma}_{2}$ and direction of principal stress $\boldsymbol{\theta}$ ) on vasculature membrane is calculated from the following equation:

$$
R=\alpha\left(\sigma_{1} \cos ^{2} \theta+\sigma_{2} \sin ^{2} \theta\right) D
$$

Here, $\boldsymbol{\alpha}$ is photoelastic coefficient, and $\boldsymbol{D}$ is thickness of vascular membrane $(\mathrm{Nu}-$ merical analysis is future work).

Presented method should be helpful for evaluating surgical procedures and the performance of medical systems, and also for various academic studies such as hemodynamic study and pathological studies.

\section{Results and Discussion}

Physical characteristics of presented model are very similar to arterial tissue. Although arterial tissue shows anisotropic nonlinear characteristics, elastic modulus of human artery normally ranges from 1 to $3 \mathrm{MPa}$, and applied silicone elastomer takes fairly close 1.9 MPa (Table 1). An this value is adjustable, by mixing silicone oil before polymerization, and it is possible to imitate sclerotic and other conditions. Friction coefficient between the surface of proposed model and LDPE (low-density polyethylene) catheter was 0.041 . It is also fairly close to the vascular value 0.039 measured between artery surface and same LDPE catheter by R.A. Caldwell et al. (Table 1).

Proposed modeling method allowed constructing thin vascular lumens, and minimally attainable diameter was $300 \mathrm{~mm}$ (geometrical error: less than $40 \mathrm{~mm}$ ). Furthermore, we realized artery-like smooth lumen surface by slightly dissolving the surface of RP lumen model with water (68 C) (Fig. 7). Here, this process less deteriorates its accuracy, as the dissolved material re-stick on the model surface. Furthermore, as shown in Fig. 8 and Fig. 9, thickness of vascular membrane is adjustable from less than $50 \mathrm{~mm}$ to more than $250 \mathrm{~mm}$ thick by adjusting withdrawing velocity in dip-coating process. Here, by layering several membranes with different thickness and different material property, it is possible, for example, to reproduce triple-layered general configuration of artery.

Consequently, proposed patient-tailored vasculature model reproduces artery-like visco-elastic deformation. Interventionalists verified the reproducibility of the viscoelastic motion, the operational feel coming from the deformation, and its usefulness for neurovascular simulations.

Since the applied elastomer and gel are transparent to visible light (transmissivity:> $88 \%$ over $10 \mathrm{~mm}$ thickness), it allowed clear recognition of inward condition, and this visibility is further improved by injecting glycerol solution (mixture of water $(44 \%)$ and glycerin $(56 \%)$, provides the same reflection index) into vascular lumens. 
Meanwhile, proposed vessel model confirmed compatible with major imaging modalities, such as CT, MR and supersonic imaging.

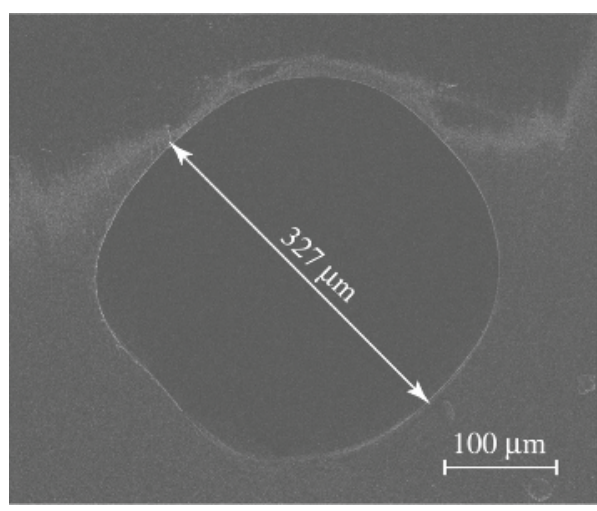

Fig. 7. SEM cross-section of fabricated vascular lumen with $300 \mu \mathrm{m}$ in diameter (modeling error was less than $40 \mu \mathrm{m}$ )

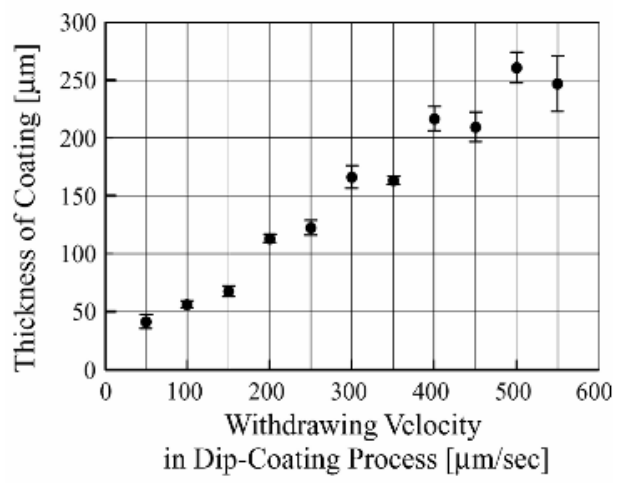

Fig. 9. Correlation between thickeness of membrane and withdrawing velocity in dipcoating fabrication process

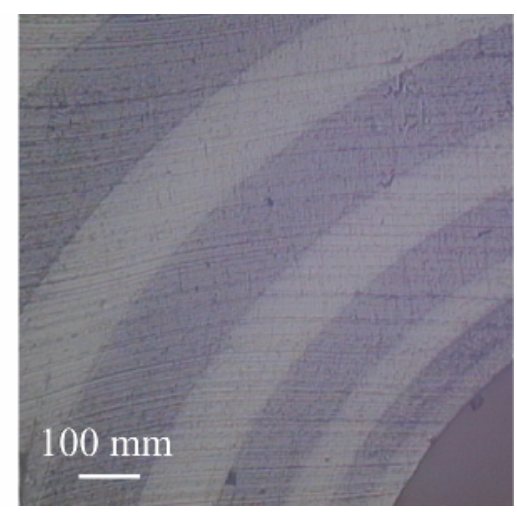

Fig. 8. Cross-section of layered silicone membrane with different wall thickness (from less than $50 \mu \mathrm{m}$ to more than $250 \mu \mathrm{m}$ )

Table 1. Comparison of material properties between vessel model and human arterial

\begin{tabular}{ccc}
\hline & $\begin{array}{c}\text { Elastic Modulus } \\
{[\mathrm{MPa}]}\end{array}$ & $\begin{array}{c}\text { Friction } \\
\text { Coefficient }\end{array}$ \\
\hline $\begin{array}{c}\text { Cerebral Arterial } \\
\text { Model } \\
\begin{array}{c}\text { Arterial Tissue } \\
\text { I } ~\end{array} \text { 3 (Carotid) }\end{array}$ & 0.031 \\
\hline
\end{tabular}

Table 2. Comparison of material properties between vessel model and human brain

\begin{tabular}{cc}
\hline & $\begin{array}{c}\text { Elastic Modulus } \\
{[\mathrm{kPa}]}\end{array}$ \\
\hline $\begin{array}{c}\text { Cerebral } \Lambda \text { rterial } \\
\text { Model }\end{array}$ & 5.0 \\
Brain Gray Matter & $6.6(\Lambda$ veraged $)$ \\
\hline
\end{tabular}

We evaluated its effectiveness for the preliminary simulation. Representative case was female, age of 72 , IC aneurysm $(15 \mathrm{~mm})$. It initially seemed wide-necked and difficult to treat by endovascular approach. In this case, we constructed a patienttailored vessel model of IC aneurysm, and tested intervention procedures within this model (Fig. 10). In practical surgery, we could lead mircocathter into IC aneurysm from its orifice locating $\mathrm{C} 2 / \mathrm{C} 3$ segment with the same manner as the preliminary 
simulation (Fig. 11). We do not confirm any coil compaction in the follow-up after 6 months in this represent case.

Cerebral aneurysms are now widely treated with endovascular approach and scrupulous diagnosis became more and more important for securing safety. However, interventional radiology is currently the only information available. In this sense, proposed patient-tailored vascular model might be very helpful for making diagnosis and preliminary surgical simulations, since it allows swift and safe treatment for respective cases.

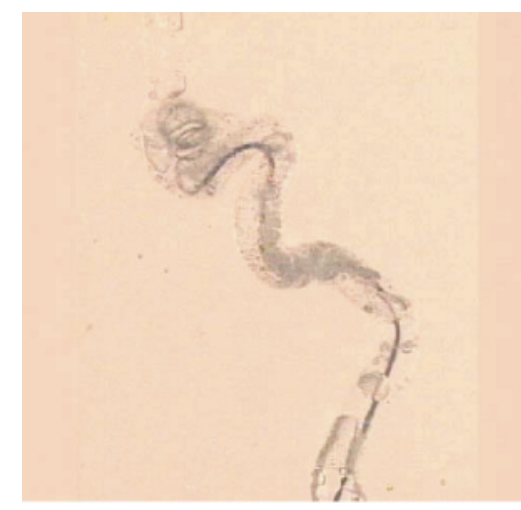

Fig. 10. Presurgical simulation (inserting microcathter and Pt. coils) for surgical planning with patient-tailored crebral arterial model

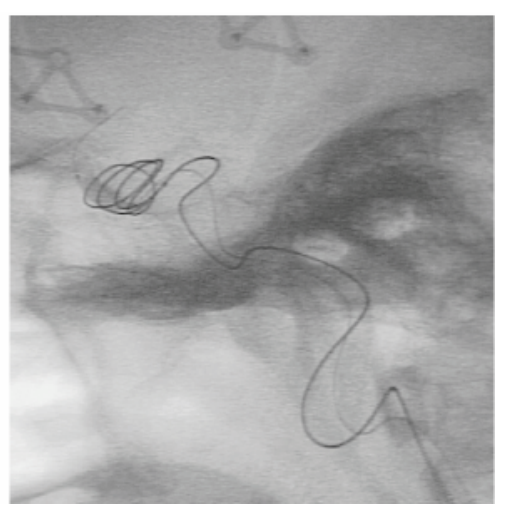

Fig. 11. DSA picture of neurovascular procedure, preliminary simulated with proposed patient-tailored vasculature model

\section{References}

1. Molyneux A, Kerr R, Stratton I, Sandercock P, et al.,: International Subarachnoid Aneurysm Trial (ISAT) of neurosurgical clipping versus endovascular coiling in 2143 patients with ruptured intracranial aneurysms: a randomised trial, Lancet, Vol. 360 (2002) 1267-74

2. P. Ng, M.S. Khangure, C.C. Phatouros, M. Bynevelt, et al.,: Endovascular Treatment of Intracranial Aneurysms With Guglielmi Detachable Coils: Analysis of Midterm Angiographic and Clinical Outcomes, Stroke, Vol. 33 (2002) 210-217

3. C.W. Kerber, C.B. Heilman: Flowdynamics in the carotid artery: 1. Preliminary observations using a transparent elastic model, Am. J. Neuroradiol, Vol. 13 (1992) 173-180

4. P. Gailloud, J.R. Pray, M. Muster, M, Piotin, et al.: An in vitro anatomic model of the human cerebral arteries with saccular arterial aneurysms, Surg Radiol Anat, Vol. 19 (1997) 119-121

5. K. Sugiu, J.B. Martin, B Jean, P. Gailloud, et al.,: Artificial Cerebral Aneurzsm Model for Medical Testing, Training, and Research", Neurol Med Chir, Vol. 43 (2003) 69-73

6. S. Ikeda, F.Arai, T.Fukuda, and M.Negoro,: An In Vitro Soft Membranous Model of Individual Human Cerebral Artery Reproduced with Visco-Elastic Behavior, Proc. 2004 IEEE ICRA, 2511-2516 